\title{
Les recherches sur les « lieux sensibles » aux États-
} Unis

\section{Sophie Body-Gendrot}

\section{(2) OpenEdition}

1 Journals

Édition électronique

URL : https://journals.openedition.org/remi/2647

DOI : $10.4000 /$ remi. 2647

ISSN : 1777-5418

Éditeur

Université de Poitiers

\section{Édition imprimée}

Date de publication : 12 décembre 2002

Pagination : 107-116

ISBN : 2-911627-32-6

ISSN : 0765-0752

Référence électronique

Sophie Body-Gendrot, « Les recherches sur les «lieux sensibles » aux États-Unis », Revue européenne des migrations internationales [En ligne], vol. $18-n^{\circ} 3$ | 2002, mis en ligne le 09 juin 2006, consulté le 14 avril 2022. URL : http://journals.openedition.org/remi/2647 ; DOI : https://doi.org/10.4000/remi.2647

Ce document a été généré automatiquement le 14 avril 2022.

(c) Université de Poitiers 


\title{
Les recherches sur les « lieux sensibles » aux États-Unis
}

\author{
Sophie Body-Gendrot
}

1 Au cours des années 1920, les sociologues de l'École de Chicago sont devenus légitimement célèbres pour leurs études sur la déviance, la délinquance juvénile et la criminalité. L'histoire de la criminalité à Chicago est marquée par les vagues successives d'immigration (Coulon, $1992: 57$ ). Les sociologues ont voulu voir dans les quartiers - et non seulement dans les individus -, des caractéristiques susceptibles d'expliquer le caractère criminogène de certains lieux. En 1929, Clifford Shaw, Frederic Zorbaugh, Leonard Cottrell et Henry McKay publient une étude sur la délinquance urbaine, Delinquency Areas, après avoir recensé les domiciles d'environ 60000 « truands, criminels et délinquants ». Ils montrent que les taux de criminalité et de délinquance sont variables d'un quartier de la ville à l'autre. Les bas quartiers situés près du centre commercial et des affaires sont les plus criminogènes. Clifford Shaw et Henry McKay (1942) développent ensuite une écologie de la délinquance et du crime et lient la croissance de la délinquance et les désordres au concept de désorganisation sociale.

2 En étudiant certaines grandes villes et en se fondant sur des calculs statistiques établissant des corrélations, des équations et des régressions linéaires présentées sous forme de cartes, ils montrent que la criminalité est associée à la structure physique de la ville. Dans leur principal ouvrage, Juvenile Delinquency and Urban Areas, (1942) ils ont identifié pauvreté, hétérogénéité ethnique et instabilité résidentielle comme variables prédictives conduisant à la délinquance. Ils ont révélé que la délinquance persistait à Chicago dans les quartiers pauvres d'immigrants, même lorsque la composition de la population changeait, comme si elle était transmise à travers les générations dans les lieux marqués par la désorganisation sociale. La corrélation entre le fort taux d'immigration dans un quartier et le taux élevé de délinquance n'entraînait pas pour autant un lien de cause à effet. «S'il ne fait pas de doute que la proportion d'étrangers et de Noirs est plus élevée dans les quartiers où la délinquance est forte, les délinquants traduits devant les tribunaux ne le sont pas parce qu'ils sont fils d'immigrés ou parce 
qu'ils sont noirs, mais pour d'autres raisons qui tiennent à la situation dans laquelle ils vivent » (Kornhauser, $1978: 163,164)$.

Pour l'École de Chicago, la délinquance n'était pas, en effet, un facteur isolé, elle était associée à de nombreux problèmes sociaux caractérisant un quartier en termes de mortalité infantile, de tuberculose, de violence domestique qui avaient une incidence sur les enfants. Profil d'un quartier, santé publique et délinquance étaient liés.

De nos jours, nombre des variables évoquées sont retenues dans les recherches l'instabilité de la population (Costanzo et al., 1996), le déplacement de la délinquance, les zones de danger (hot spots) (Block et Block, 1984) - liant environnement spatial et délinquance (Sampson et Groves, 1989). Or il convient d'examiner cette hypothèse selon diverses perspectives (Body Gendrot, 1998; 2000). De quelles connaissances disposons-nous lorsque nous percevons l'environnement urbain comme dangereux ? En quoi la littérature sur le sujet peut-elle nous être utile? Les variables sont-elles comparables d'une ville à l'autre? Les variations correspondent-elles partout à des différences socio-économiques et raciales/ethniques? Un bref examen des thèses en présence permet d'en relativiser l'importance.

Le concept de désorganisation sociale

On doit à $\mathrm{W}$. Thomas, le thème central de la désorganisation individuelle et collective dans les quartiers qui correspond à un déclin de l'influence des règles sociales sur les individus, à un affaiblissement des valeurs collectives et à une valorisation des pratiques individuelles. Ce phénomène s'amplifie lorsqu'une société connaît des mutations rapides. À Chicago, ville de croissance rapide, il prend une tournure spectaculaire. La désorganisation familiale d'immigrants et d'ouvriers saisonniers entraîne paupérisation, délinquance juvénile et violence dans les quartiers.

C. Shaw et H. Mckay (1942) avancent que la violence des quartiers marqués par la pauvreté, par une forte diversité ethnique et par une grande mobilité résidentielle résulte d'un manque d'organisation sociale, c'est-à-dire d'une incapacité à unir le quartier autour de valeurs communes et à y maintenir des contrôles sociaux efficaces. L'absence de liens avec une famille et avec des amis se traduit par des carences associatives, et une non-prise en charge des problèmes collectifs créés, par exemple, par des adolescents oisifs. Nul ne s'autorise à intervenir pour le bien-être général. Toutefois pour W.F. Whyte (1943), les communautés semblent désorganisées par rapport aux critères de la société américaine globale et de ses institutions, mais elles se réorganisent selon des critères spécifiques, complexes, structurés à partir de systèmes hiérarchisés fondés sur des obligations réciproques. L'américanisation de masse passe par l'appartenance à des mondes ethniques qui se modifient progressivement et concourent à l'adaptation. La délinquance se produit en partie lorsque contradictions et pressions en vue de conformisme normatif affaiblissent trop tôt les communautés d'origine et « démoralisent » les individus.

La nouvelle recherche écologique

7 Elle s'attache à l'étude de villes (Harries, 1996) ou de quartiers (Katzman, 1981) dont les variables peuvent expliquer l'intensité ou non des activités délinquantes. Les données du recensement servent de base de départ (niveau socio-économique, taux de familles monoparentales, race) mais sont insuffisantes. Elles doivent être complétées par des entretiens individuels (Fagan et Davis, 1998). À Chicago, Richard Block (1997) a trouvé que dans certains quartiers peuplés à plus de $75 \%$ par des Noirs, le taux d'homicide était de 51 pour 100000 alors que dans les quartiers noirs à $25 \%$, il était de 6 pour 
100 000. Pourtant, c'est moins la race que deux autres variables qui expliquaient ce phénomène de criminalité élevée : d'une part le pourcentage de migrants du sud dans un quartier, région de tradition violente et d'autre part, la présence spatiale rapprochée de familles riches et de familles pauvres. Cette coexistence des inégalités, les frustrations et les tentations qu'elle engendrait expliquait, selon Block, des différences de $56 \%$ pour les homicides et de près de $40 \%$ pour les vols et les attaques.

D'autres études confirment que ce sont moins les jeunes gens défavorisés dans les quartiers pauvres qui sont le plus poussés à la délinquance que les jeunes gens issus de familles défavorisées vivant dans des quartiers aisés (Johnstone, 1978). Pour Sampson et Groves (1989), en incluant toutes les variables pertinentes dans une étude macrosociologique des quartiers (urbanisation, organisation des habitants, diversité ethnique, état des familles), on s'aperçoit que le statut socio-économique et la diversité ethnique viennent en position secondaire. Ce sont parfois les caractéristiques des quartiers adjacents qui sont explicatives de la délinquance d'un quartier en matière de vols (Katzman, 1981). Harries (1996) a noté incidemment une corrélation au Texas entre la température extérieure et les attaques dans les quartiers pauvres. L'absence d'air conditionné accentue l'énervement et la consommation de boissons alcoolisées les jours de grande chaleur dans ces quartiers. Inversement, on a noté que lorsque les hivers sont très rigoureux, la délinquance baisse comme le thermomètre.

Menées ethnographiquement à l'échelon des pâtés de maisons à Cleveland et à San Francisco, des recherches suggèrent que la criminalité est liée à la sur-représentation des individus isolés et des grands ensembles surpeuplés dans lesquels les gens ne se connaissent pas. Les vols plus que les délits violents sont corrélés à ce type d'environnement (Roncek et al.,1981). Mais d'autres études infirment ces observations. Selon, une recherche portant sur 3729 adolescents dans quatre villes, la richesse ou la pauvreté d'un quartier ont moins d'effets sur la criminalité que les caractéristiques individuelles des personnes (Gottfredson, Hirshi 1990). Qu'en déduire?

Robert Sampson et son équipe (1989) ont mené des analyses à partir du National Crime Survey, étude de victimisation conduite auprès de 100000 individus. Il en ressort que les inégalités socio-économiques et les manques influent moins sur la délinquance que, par ordre décroissant: la structure familiale, la densité de l'habitat, la mobilité résidentielle (le dépeuplement), la composition raciale.

11 Selon les conclusions de cette étude, la structure des familles (monoparentale ou non) était cinq fois plus explicative que la composition raciale d'un quartier. La structure des familles et la densité de l'habitat avaient une valeur prédictive sur le vol tandis que le dépeuplement et la transformation du quartier sont les plus explicatifs en termes de variable. La race n'était importante que par rapport à la structure familiale monoparentale.

12 Dans une autre étude (1997), le même auteur a établi que l'ensemble des variables suivantes - pauvreté, pourcentage de Noirs, densité de l'habitat, urbanisation et âge des victimes - ont une incidence sur les taux de vols et de délits violents. L'âge des victimes, le taux d'urbanisation et la densité de l'habitat expliquent la plus grande partie des variations, à l'inverse de la pauvreté et de la composition raciale, contrairement aux stéréotypes répandus. Toutefois, seule l'interaction de toutes les variables rend compte de la complexité du phénomène.

La difficulté s'accroît lorsque les décideurs cherchent à être normatifs. Pour s'en tenir à la recherche précédente, il faudrait donc réduire le nombre de familles 
monoparentales, freiner les déménagements, diminuer la pauvreté et la densité de l'habitat et de l'urbanisation, accroître l'instruction, le contrôle social et les réseaux de sociabilité pour réduire la délinquance.

Autres types de recherches urbainesLes espaces à défendre

14 Jane Jacobs (1961) et Oscar Newman (1973) ont développé l'idée que l'environnement a un effet sur la fréquence des contacts que les habitants ont entre eux et sur le contrôle social interne du voisinage (Perkins et al., 1993 ; Greenberg et Rohe, 1984). Du point de vue de l'espace plutôt que des individus, la théorie de "l'occasion" démontre que l'absence de systèmes d'alarmes, la facilité d'accès, un mauvais éclairage, la coexistence d'impasses et d'allées sombres, la circulation automobile, les parkings et les boutiques (objets de convoitise) accroissent le potentiel de délinquance. Forte est la probabilité qu'un délit se produise dans un lieu dépourvu de protection (gardien, policier, conducteur d'autobus, alarmes, chiens, etc.) où victimes et délinquants potentiels coexistent (Felson et Cohen, 1980). Les activités routinières des victimes et des délinquants potentiels ont une valeur prédictive. Les délinquants attendent que des occasions favorables se présentent dans un environnement qu'ils connaissent (maisons vides; personnes sur des trajets exposés ). Entre 1960 et 1970, il a été démontré qu'un accroissement de $31 \%$ de femmes au travail et de $28 \%$ de voitures par habitant s'est traduit par une corrélation avec la hausse de la délinquance.

Jane Jacobs a pensé néanmoins qu'en créant un espace ouvert à la sociabilité, avec des bancs et des placettes où les gens viendraient s'asseoir, avec de larges trottoirs où joueraient les enfants et un mixte de commerces et d'habitations, le flux constant de gens produirait une surveillance informelle des lieux.

Oscar Newman (1972), quant à lui, a préconisé la pose de barrières physiques (grilles, murs) et symboliques (buissons, éclairage) qui agiraient comme des marqueurs territoriaux sur l'espace. Il a suggéré de remplacer les grands ensembles par de petits immeubles pour faciliter les interactions et l'identification des gens et des lieux.

Les manques relatifs (relative deprivation)

Dans l'optique privilégiant la responsabilité des individus, dans les villes et les quartiers c'est la proximité d'une population à faibles revenus et d'une population plus aisée qui génère la délinquance. La frustration émotionnelle provoquée par la juxtaposition de ces populations, l'impossibilité de se procurer des biens de consommation perçus comme indispensables créent "des animosités latentes, porteuses de violence" (Sampson, 1989). Cette théorie qu'affectionnent les journalistes est moins prise au sérieux que les précédentes.

Le choix rationnel

18 Selon la théorie du choix rationnel, les délinquants feraient une estimation des risques et des coûts de leurs actions. Ils disposeraient de toute l'information nécessaire pour décider en toute connaissance de cause si le jeu en vaut la chandelle. Cette théorie difficilement crédible a été remplacée par celle de la rationalité partielle qui inclut les limites imposées par une information incomplète (Akers, 1997).

La mobilité des délinquants

En 1986, Alicia Rand a étudié la relation entre le lieu d'un délit, le quartier de résidence du délinquant et celui de la victime et elle a pu établir que $30 \%$ des délinquants agissaient dans leur quartier (soit dans la même unité de recensement), proportion s'élevant à $53 \%$ dans les cas de viols et d'homicides, à $14 \%$ pour les vols de biens, à $23 \%$ pour les vols d'automobile au caractère plus planifié (Rand, 1986). Il avait déjà été 
montré à Milwaukee qu'à partir du ghetto noir, un grand nombre de délinquants se rendaient occasionnellement dans d'autres zones pour voler et non pour cambrioler alors que les délinquants habitants de ces zones ne se rendaient pas dans le ghetto noir pour y commettre des méfaits. Mais la validité de l'analyse a été réduite par la nonprise en compte des tailles de population et des quartiers concernés, de l'environnement physique et des conditions socio-économiques.

Costanzo et al. (1986) se sont intéressés à la direction du trajet suivi par les délinquants. Ils ont observé que ceux-ci prenaient les mêmes trajets pour commettre les mêmes types de délits, sans doute en raison d'une même information. Or à partir de faits élucidés, cette information pouvait être utilisée en retour par la police de manière à remonter des filières de délinquance. D’autres chercheurs ont précisé ces données : ils ont montré, par exemple, que les cambrioleurs se déplacent plus loin que les violeurs et que les meurtriers (Pyle, 1976) et que les délinquants de plus de vingt ans bougent deux fois plus que les autres, les femmes plus que les hommes, les cambrioleurs blancs trois fois plus que les cambrioleurs noirs (Nichols, 1980 ; Philipps, 1980). Mais il convient de relativiser ces informations recueillies auprès des individus arrêtés (Rhodes, 1981).

L'étude des lieux sensibles

La recherche micro appelée aussi criminologie environnementale ou encore prévention situationnelle désagrège le voisinage en petites unités - pâtés de maisons et lieux de délinquance (Sherman et al., 1989 ; Felson et Cohen, 1980) de manière à établir de fines corrélations entre espace et population par rapport à la variation des taux de délinquance. Le thème des zones sensibles (hot spots) permet d'accoler marqueurs spatiaux (entrées de métro, bars, grands ensembles) et délinquance (Block, 1997). La taille de ces zones varie depuis l'emplacement à proximité d'une caisse automatique de banque jusqu'aux entrées et sorties d'un centre commercial.

On peut distinguer les lieux (places), où se produisent des faits divers (aux arrêts d'autobus et aux coins des rues) et les zones (spaces) qui intègrent à la fois les lieux et les faits divers (unités de recensement, circonscription de police, etc). Une relation réciproque et interactive se produit entre les lieux et les zones adjacentes selon la manière dont évolue la pauvreté d'un quartier, selon qu'il est isolé ou inséré dans un plus vaste ensemble de quartiers pauvres (Jargowsky, 1996), selon que les services de base (pompiers, police, centres de traitement de la toxicomanie) sont ou non absents. Sherman et al. (1989) ont analysé 323980 appels police-secours sur une année et ont établi qu'ils correspondaient à $3,3 \%$ des adresses et des coins de rue à Minneapolis. Tous les vols étaient concentrés sur 2,2 \% des lieux, les vols de voiture sur 2,7 \% et les viols, $1,2 \%$, mais des variations existaient au sein de ces lieux. Pour confirmer ces informations, une recherche sur les lieux non sensibles (cold spots) s'imposerait.

Y a-t-il d'avantage de délinquance dans les logements publics, comme le laisse entendre le discours commun ? Les raids opérés par la police, la publicité donnée à quelques faits divers tendent à accréditer ces stéréotypes. À une ou deux exceptions près (Huttman et al., 1991), il n'existe pratiquement pas de recherches sur le crime et la violence dans les grands ensembles aux États-Unis (Fagan et Davies, 1998:2). Or les logements sociaux sont loin d'être uniformes, comme le montrent les statistiques données plus haut. Les taux de délinquance varient en conséquence (Williams et Kornblum, 1985). De plus, ces villages urbains ne sont pas statiques, ils évoluent et la délinquance suit ces mutations. En dix ans à Harlem, dans un seul grand ensemble sur les quatre étudiés par Kornblum on a enregistré des taux consistants de criminalité. Enfin, alors que le nombre de 
plaintes et d'arrestations donne l'impression que le taux de criminalité est élevé, rapporté au nombre de locataires officieux, il l'est souvent moins que dans les zones adjacentes. Or la question de la contagion ou de la proximité de zones dangereuses sur l'espace du logement social est mal connue.

Pour Jeff Fagan, directeur du centre d'étude de la violence et de la prévention à Columbia University, l'étude qu'il a menée avec son équipe sur le logement social du Bronx tend à montrer que la diffusion des actes violents (meurtres, viols, vols avec armes) se fait à partir de certains ensembles de logements sociaux sur les zones adjacentes, à l'exception des attaques à main armée dont les effets opèrent dans les deux sens (Fagan et Davies, $1998: 3$ ).

Pourquoi certains "ghettos verticaux" sont-ils plus dangereux que d'autres? Les caractéristiques de leur population et la dynamique des interactions avec les zones adjacentes fournissent-elles la clé de l'explication ? Rien n'est moins évident. Les études sur les «zones interstitielles" autour des logements sociaux sont difficiles à mener. Mais de manière générale, les logements sociaux sont localisés dans des zones de détresse économique et sociale qui, dans l'optique américaine, n'ont pas eu assez de ressources politiques ou de pouvoir de sanction pour s'opposer à la construction de grands ensembles susceptibles d'attirer les ménages les plus pauvres de la ville (BodyGendrot, 2000 ; 2001). Cette attitude témoigne d'une mentalité « bien américaine » : les quartiers sont en compétition. Les plus forts parviennent à se passer des dispositifs indésirables - logements sociaux - que les plus faibles sont bien obligés d'accueillir. Il en est de même sur le plan social : la malchance des plus pauvres veut qu'ils aient à habiter à proximité des individus les plus délinquants qui exercent sur eux des actions prédatrices. Par exemple, dans certains de ces grands ensembles du Bronx, rappelle J. Fagan, le jour où les chèques de l'aide sociale arrivent, (appelé dérisoirement « la fête des mères »), les ambulances sont là aussi. Les individus paupérisés se victimisent les uns les autres ou alors quittent le quartier dès qu'ils le peuvent. On peut d'ailleurs faire ici l'observation qu'alors que nos politiques de la ville territorialisent les mesures et travaillent sur les "quartiers sensibles", les programmes américains aident à la mobilité des populations qui «votent avec leurs pieds " pour échapper aux problèmes urbains dès qu'elles en ont l'occasion. Le ghetto historique de Chicago a perdu la moitié de sa population depuis les années 1970. La raison en est simple : les appartements des plus pauvres sont plus fréquemment cambriolés, ils sont moins bien protégés et plus commodes d'accès que les appartements dans les quartiers aisés qui bénéficient par ailleurs de technologies de surveillance privées. Cependant les zones sont loin d'être uniformes et les processus que l'on peut y observer peuvent être diamétralement opposés.

26 À Chicago, Richard Block (1997) explique qu'il existe des points dangereux dans des zones non sensibles et que l'inverse est vrai aussi. Par exemple, une taverne à proximité d'un métro ou d'un parking constitue un lieu dangereux. Les consommateurs s'enivrent, ils ont de l'argent, c'est la nuit, ils peuvent être attaqués et les assaillants s'enfuient par le métro le plus proche ou par le parking. Dans la $26^{\text {ème }}$ circonscription de la police, par exemple, 43000 vols se sont produits en deux ans. Ce district, plus vulnérable que d'autres, ne compte pas moins de 80 magasins de vins et spiritueux dans lesquels il est possible de consommer. Ils se trouvent tout au long de la ligne de métro aérien de Chicago. Les stations les plus propices à la délinquance sont celles dans lesquelles se croisent deux lignes de métro. Les vols se produisent fréquemment entre 
minuit et deux heures du matin, quand les clients sont ivres, à la fermeture des magasins de vin, ce qui montre le caractère encore très prégnant des études de Clifford Shaw menées dans les années 1930. Les stations faisant la jonction entre la ville et les lignes de banlieue, soit entre des lieux où la consommation d'alcool est interdite dans les lieux publics depuis la Prohibition et les quartiers pauvres de la ville, sont prisées par les délinquants, le métro leur permettant de s'enfuir rapidement, une fois leurs délits accomplis.

L'utilisation de l'informatique

Si les délits ne se font pas au hasard, s'ils tendent à se produire dans des lieux probables, des modèles mathématiques peuvent alors être élaborés entre lieux de résidence des délinquants, lieux de délits (hot spots) et zones-tampon intermédiaires. Le Criminal Geographic Targeting (CGT) remplit cette fonction. À partir des informations tirées d'une demi-douzaine de délits, le CGT peut réduire une zone d'investigation pour la police de $90 \%$, lui faire gagner du temps et rendre plus efficaces ses ressources, grâce aux analyses fournies par les GIS (Geographic Information Systems) dont toutes les grandes villes américaines sont pourvues.

Cet appareillage hautement technologique interpelle les chercheurs : ne lui accorde-ton pas trop d'importance dans une optique de prévention situationnelle de la délinquance? L'information peut-elle efficacement entraver les desseins des délinquants? Ne réagissent-ils pas en retour aux innovations informatiques de la police en s'adaptant continuellement? La présence accrue de la police dans les lieux de forte délinquance ne les incite-t-elle pas à se déplacer vers d'autres quartiers? Certaines études indiquent que la corrélation est négligeable et inexistante pour les délits violents mais il est certain que d'autres recherches s'avèrent nécessaires pour cerner le phénomène.

\section{BIBLIOGRAPHIE}

AKERS Roger (1997) Criminological Theories : Introducing and Evaluation, Roxbury Publishing Company, Los Angeles.

BLOCK RICHARD (1997) Risky Places in Chicago and The Bronx. Robbery in the Environs of Rapid Transit Stations, Working paper.

BLOCK Carolyn and BLOCK Richard (1984) Crime Definition, Crime Measurement, and Victim Surveys, Journal of social Issues 40 (1), pp. 137-160.

BODY-GENDROT Sophie (1998) Les villes face à l'insécurité, Bayard Editions, Paris.

BODY-GENDROT Sophie (2000) The social control of cities? A Comparative Perspective, Blackwell, Oxford.

BODY-GENDROT Sophie (2001) Villes : la fin de la violence? Presses de Sciences Po, Paris. 
COSTANZO C., HALPERIN W. and GALE N. (1986) Criminal Mobility and the Directional Component in Journeys to Crime in Figlio R., Hakim S. and Rengert G. eds, Metropolitan Crime Patterns, Criminal Justice Press, Monsey, NY.

COULON Alain (1992) L'école de Chicago, Paris, PUF.

ECK J. and WEISBURG D. (1995) Crime Places in Crime Theory in J. Eck and D. Weisburg eds., Crime and Place, Criminal Justice Press, Monsey, NY.

FAGAN Jeff and DAVIS Garth (1998) « The social context and Functions of Adolescent Violence » in D. Elliott and. B. Hamburg eds., Violence in American Schools, Cambridge University Press, Cambridge.

FAGAN Jeff (1997) The Comparative Advantage of Juvenile Versus Criminal Court Sanctions on Recidivism among Adolescent Felony Offenders, Law and Policy, 18, 1-2, Jan-April 1996, pp. 77-114.

FELSON M. and COHEN L. (1980) « Human Ecology and Crime : A Routine Activity Approach », Human Ecology, 8 (4) pp. 389-406.

GOTTFREDSON Michael and HIRSHI Travis (1990) A General Theory of Crime, Stanford Univ. press, Standford.

GREENBERG Stanley and RHOE William (1984) « Neighborhood Design and Crime : A Test of Two Perspectives ", Journal of the American Planning Association, 50, pp. 48-61.

HARRIES K. (1996) «Cities and Crime », Criminology, 14 (3), pp. 369-386.

HUTTMAN Elizabeth ed. (1991) Urban Housing. Segregation of Urban Minorities in Western Europe and in the United States, Duke University Press, Durham.

JACOBS Jane (1961) The Life and Death of American Cities, Penguin,Hardmonsworth, London.

JARGOWSKY Paul (1996) Poverty and Place: Ghettoes, Barrios, and the American City, Russell Sage Foundation, New York

JOHNSTONE James (1978) « Social Class, Social Areas and Delinquency », Sociology and Social Research, 63, pp. 49-72.

KATZMAN M. (1981) The Supply of Criminals : A Geo-Economic Examination, in S. Hakim and G. Rengert eds., Crime Spillover, Sage Publication, Beverley Hills.

KORNHAUSER Ruth (1978) Social Sources of Delinquency, Univ, of Chicago Press, Chicago.

NEWMAN Oscar (1972) Defensible Space, Crime Prevention Through Urban Design, Macmillan, New York.

NICHOLS William (1980) «Mental Maps, Social Characteristics and Criminal Mobility », in D. Georges-Abayie and K. Harries eds., Crime : A Spatial Perspective, Columbia University Press, New York.

PARK Robert, BURGESS Ernest and McKENZIE Robert (1925) The City, Univ, of Chicago Press, Chicago, reprint. 1967.

PERKINS D., WANDERSMAN A,. RICH R. and TAYLOR, R. (1993), « The Physical Environment of Street Crime : Defensible Space, Territoriality and Incivilities », Journal of Environmental Psychology, 13, pp. 29-49.

PHILIPPS P. (1980) Characteristics and Typology of the Journey to Crime, in D. Georges-Abayie and K. Harries eds., op.cit. 
PYLE George (1976) « Spatial and Temporal Aspects of Crime in Cleveland, Ohio », American Behavioral Scientist, 20 (2), pp. 175-198.

RAND A. (1986) Mobility Triangles, in R. Figlio, S. Hakim and G. Rengert eds., Metropolitan Crime Patterns. Criminal Justice Press, Monsey, NY.

RHODES R.A. (1981) Control and Power in Central-Local relations, Gower, Farnborough.

RONCEK, D., BELL, R. and FRANCIK J. (1981) Housing Projects and Crime : Testing a Proximity Hypothesis, Social Problems, 29 (2), pp. 151-166.

SAMPSON Robert, RAUDENBUSH Stephen and FELTON Earls (1997) « Neighborhoods and Violent Crime : A Multilevel Study of Collective Efficacy », Science 277, August, 9, pp. 18-924

SAMPSON Robert and GROVES W. Byron (1989) « Community Structure and Crime : Testing Social Disorganization Theory », American Journal of Sociology, 94, pp. 774-802.

SHAW Clifford (1930) The Jack-Roller. A Delinquent boy's own story, The University of Chicago Press, Chicago.

SHAW Clifford and McKAY Henry (1942) Juvenile Delinquency and Urban Areas. University of Chicago Press, Chicago.

SHAW Clifford, ZORBAUGH Frederic, McKAY Henry and COTTRELL Leonard (1929) Delinquency Areas : a Study of Geographical Distribution of Schools Truants, Juvenile Delinquents and Adult Offenders in Chicago, University of Chicago Press, Chicago.

SHERMAN Lawrence, GARTIN P. and BUERGER M. (1989) « Hot spots of Predatory Crime : Routine Activities and the Criminology of Place », Criminology, 27 (1), pp. 27-55.

WEISBURD D., MAHER L. and SHERMAN Lawrence (1992) Contrasting Crime General Crime Specific Theory: The Case of Hot Spots of Crime, in F. Adler and W. Laufer eds., Advances in Criminological Theory, vol. 4, Transaction Publishers, New Brunswick, NJ.

WILLIAMS Terry and KORNBLUM William (1985) Growing Up Poor, Lexington Books, Lexington, Ma.

WHYTE William (1943) Street Corner Society. The social structure of an Italian Slum, University of Chicago Press, Chicago.

\section{INDEX}

Index géographique : États-Unis

Mots-clés : espace urbain, sociologie urbaine

\section{AUTEUR}

\section{SOPHIE BODY-GENDROT}

Professeur des Universités, Sorbonne - Paris IV, chercheur au CESDIP (CNRS), 43 Bd Vauban, 78280 Guyancourt. 